\title{
Cross-gender perceptions of facial attributes and their relation to attractiveness: Do we see them differently than they see us?
}

\author{
JO ELLEN MEERDINK, CALVIN P. GARBIN, and DANIEL W. LEGER \\ University of Nebraska, Lincoln, Nebraska
}

\begin{abstract}
Examination of perceptions of human facial attributes revealed that individual attributes are similarly perceived by males and females. However, patterns of attribute interrelationships differ as a function of gender of the face. Undergraduate students $(N=280)$ rated pictures of 40 male or female Caucasians on 12 physical attributes (e.g., nose size, face width) and overall attractiveness. The four sets of attribute ratings (defined by rater gender and picture gender) were submitted to principal components analyses, and five-factor solutions were found for each condition (accounting for about $76 \%$ of the variance). Comparisons of the four component solutions using confirmatory factor procedures revealed that male and female raters share one factor structure when rating photographs of female faces and another factor structure when rating photographs of male faces. Multiple regression analyses revealed that the patterns of attribute interrelationships were not "perceptual units" in the perception of attractiveness, and that different "rules" are used to assess the attractiveness of male and female stimuli faces. The importance of these results for models of facial attractiveness and interfacial similarity judgments are discussed.
\end{abstract}

Physical attractiveness is one of the major determinants of interpersonal attraction among Americans (Berscheid $\&$ Walster, 1974). Buss (1985) reported that physical attractiveness ranked third for males and sixth for females on a list of characteristics commonly sought in a mate. Walster, Aronson, Abrahams, and Rottman (1966) conducted a field study in which individuals were randomly paired with one another at a "computer dance." They concluded that the only important determinant of an individual's liking for his date was the date's physical attractiveness. Finally, Murstein (1972), investigating the idea that people select marital partners of similar physical attractiveness to themselves, concluded that physical attractiveness (both subjectively experienced and objectively measured) operates by what he calls exchange-market rules. That is, individuals with equal market value for physical attractiveness are more likely to associate in an intimate relationship than are individuals with disparate values.

Although the evidence is overwhelming that physical attractiveness is a major factor in many aspects of everyday life, including the preference for and selection of a mate, we seem to lack an adequate definition of attractiveness. Many studies have been conducted that have helped to establish the importance of physical attractiveness, but few have involved its assessment. Many researchers have concentrated on facial attractiveness, but again, few (e.g., Cunningham, 1986) have attempted to define how perceptions of physiognomy lead to the "per-

Correspondence should be addressed to Jo Ellen Meerdink, 209 Burnett Hall, Department of Psychology, University of Nebraska, Lincoln, NE 68588-0308. ception" of attractiveness. Many researchers have instead relied on the consensus of a panel of judges as to which faces were attractive and which were not. We feel that, in order to study the relationship between facial features and perceived attractiveness, one must determine whether or not people perceive facial attributes in the same manner, and whether or not they share the same perceived attribute interrelationships. Only when this has been done can one consider how the perceptions of attributes and their interrelationships translate into attractiveness. Finally, one can examine the ways in which the three concepts of physiognomy, attractiveness, and similarity influence preferences-Do people prefer certain combinations of attributes, and how much of that preference is influenced by similarity to themselves?

An obvious confounding factor in studies of attractiveness is race. Malpass and Kravitz (1969) found that white faces were more discriminable than black faces, and that black and white subjects had higher acuity for faces of their own race. Shepherd, Deregowski, and Ellis (1974) found similar results using both European (white) and African stimuli and subjects. Subjects were superior in recognizing faces from their own race. Investigating the assessment of attractiveness, rather than facial recognition, Bernstein, Lin, and McClellan (1982) worked with subjects from three ethnic groups (white and Chinese in one experiment, white and black in a second experiment). Their results indicated that "the various ethnic groups do use slightly different standards of attractiveness, but they are all capable of applying these rules to other groups as well as to their own" (Bernstein et al., 1982, p. 501).

Cunningham (1986), who used measurements of facial features (e.g., length of face measured from the hairline 
to the base of the chin) in a multiple regression analysis to predict physical attractiveness, found that certain features produced a significant prediction equation. Cunningham's male subjects rated pictures of female faces on an attractiveness scale from 1 to 6 and then, using physical measurements of the faces, examined the types of features that were present on the faces considered to be attractive. The following features were significantly correlated with attractiveness: eye height (.50), eye width (.41), separation of eyes $(.29)$, nostril width $(.33)$, nose area $(-.34)$, chin length $(-.38)$, cheekbone width $(.58)$, cheek width ( -.47$)$, eyebrow height (.46), pupil width (.46), and smile width (.53). Cunningham then used these facial feature measurements to predict attractiveness ratings for the pictures. A significant multiple regression equation resulted $(R=.77)$. The features measured by Cunningham were grouped into three sets: neonate $(R=.34$ with attractiveness independent of the other two groups), mature $(R=.25)$, and expressive $(R=.23)$. Each of these groups also produced a significant regression equation ( $R=.63, R=.61$, and $R=.63$, respectively). Cunningham found that the optimal regression analysis used a combination of features from each of the three groups: eye height, nose area (neonate), narrow cheek width (mature), and smile width (expressive). This regression equation was found to be as effective $(R=.73)$ as the full set of predictors, accounting for $52.5 \%$ of the variance in mean attractiveness ratings.

This type of analysis may or may not help one understand the perceptions and judgments that are part of attractiveness ratings. First, the approach assumes a strict relationship between physical measures of facial attributes and a person's perceptions of those attributes. Second, being a nomothetic approach, it assumes that there are no individual or group differences in the perceptions of facial features. The first consideration leads to the suggestion that more representative models of attractiveness might result if the models were based on attribute perceptions rather than physical measures (cf. Monahan \& Lockhead, 1970, for a parallel example within the shape perception literature). The use of perception-based predictors forces the consideration of group and/or individual differences of these perceptions.

Additional concerns are raised by a careful consideration of the "makeup" of prediction models of this type. The relative importance of any predictor attribute to the criterion of physical attractiveness has two sources within a multiple regression model: (1) the strength of the relationship between that attribute and the criterion and (2) the pattern of interrelationships among the attributes. Taken together with the issues of measured as opposed to perceived attributes, these considerations suggest the importance of examining two potential sources of different "attractiveness rules." The first consists of group differences in the perceptions of individual facial attributes; the second consists of group differences in the perceived interrelationships of these individual facial attributes. Answers to these questions about facial feature perception are an important prelude to attempts to identify the "rules" that people use to combine facial features into attractiveness judgments and to look for individual and group differences in these "rules."

The present study was conducted to investigate two issues. The first issue was whether or not males and females have the same perceptions of individual facial attributes and patterns of interrelationships among 12 attributes (e.g., eye size, face shape, hair length; see the Method section). The second issue was which of these 12 facial features were significant predictors of attractiveness ratings. The attributes rated by the subjects were selected as the facial features most often mentioned in the attractiveness literature.

Of principle interest were the cross-gender correlations, factor structures, and attribute models of attractiveness. The same-gender data were collected, as will be discussed below, to provide an important basis for comparison. Data were collected under four conditions, in which males and females made judgments of same-gender as opposed to opposite-gender faces. The data were collected in this manner to assess which of four competing hypotheses would best describe the patterns of similarities and differences in the perceived interrelationships (factor patterns) of the facial features among the groups. First, there might be equivalent factor structures for all four groups. That is, all raters, regardless of the gender of the subject or the face being rated, might share the same perceived patterns of interrelationships among the 12 facial attributes. Second, each combination of rater gender and face gender might yield a different factor structure. Third, two different factor structures might exist that were dependent on picture gender, but not on the rater's gender. Fourth, two different factor structures might exist that were dependent on rater gender, not on the gender of the face being rated. Other hypotheses are possible; however, these four were the most important to examine initially.

\section{METHOD}

\section{Subjects and Design}

The data for this study were provided by 280 introductory psychology students with a mean age of 19.2 years, who participated to fulfill a course requirement. The design was a 2 (rater gender) $\times 2$ (picture gender) factorial, with 70 subjects in each condition. Data from an additional 24 subjects were excluded from analysis, due to failure to follow instructions or to complete the procedure.

\section{Materials and Procedure}

Eighty black-and-white stimulus pictures (40 males and $40 \mathrm{fe}$ males, all Caucasian) were randomly selected from a college yearbook, screening for unusual facial expressions, hairstyles, clothing, eyeglasses, or facial hair, as well as for pictures that did not reproduce well. The yearbook portraits were enlarged to $3 \times 5$ in., using a photographic copier. Sets of the $\mathbf{4 0}$ same-gender pictures were laminated, four to a page.

The subjects rated each picture on each of 12 attributes, using 9-point rating scales (e.g., low-high cheek position, light-dark eye color). All pictures were rated on one attribute before the next attribute was considered, and the subjects rated the attributes in different orders. The attributes were eye size (small-large), eye spacing 
Table 1

Mean Attribute Values, with Standard Deviations and Skewness, from Female and Male Ratings of Male Pictures

\begin{tabular}{|c|c|c|c|c|c|c|}
\hline \multirow[b]{3}{*}{ Attributes } & \multicolumn{6}{|c|}{ Gender of Rater } \\
\hline & \multicolumn{3}{|c|}{ Male } & \multicolumn{3}{|c|}{ Female } \\
\hline & $M$ & $S D$ & Skewness & $M$ & $S D$ & Skewness \\
\hline Hair length & 4.41 & 1.71 & .38 & 4.77 & 1.65 & .34 \\
\hline Eye spacing & 4.97 & 1.01 & -.46 & 4.99 & 1.65 & -.31 \\
\hline Nose size & 5.43 & .89 & .46 & 5.19 & .91 & .18 \\
\hline Eye size & 5.13 & 1.82 & -.15 & 5.11 & 1.59 & -.22 \\
\hline Hair color & 5.83 & 1.89 & -.74 & 5.78 & 1.83 & -.68 \\
\hline Face length & 5.18 & 1.35 & .48 & 5.23 & 1.12 & .79 \\
\hline Mouth size & 5.16 & 1.52 & -.17 & 5.31 & 1.45 & -.34 \\
\hline Eyebrow shape & 4.92 & 1.72 & -.16 & 4.85 & 1.61 & -.31 \\
\hline Face width & 4.90 & 1.32 & .23 & 5.07 & 1.24 & .27 \\
\hline Cheek position & 5.37 & .97 & -.17 & 5.40 & .88 & -.30 \\
\hline Face shape & 4.94 & .96 & -.58 & 5.03 & 1.02 & -.52 \\
\hline Eye color & 5.53 & 1.85 & -.63 & 5.47 & 1.82 & -.59 \\
\hline
\end{tabular}

Table 2

Mean Attribute Values, with Standard Deviations and Skewness, from Female and Male Ratings of Female Pictures

\begin{tabular}{|c|c|c|c|c|c|c|}
\hline \multirow[b]{3}{*}{ Attributes } & \multicolumn{6}{|c|}{ Gender of Rater } \\
\hline & \multicolumn{3}{|c|}{ Male } & \multicolumn{3}{|c|}{ Female } \\
\hline & $M$ & $S D$ & Skewness & $M$ & $S D$ & Skewness \\
\hline Hair length & 5.26 & 2.37 & -.21 & 5.61 & 2.84 & -.34 \\
\hline Eye spacing & 5.32 & 1.06 & .07 & 5.01 & .97 & .00 \\
\hline Nose size & 5.08 & 1.12 & -.02 & 5.03 & 1.05 & .13 \\
\hline Eye size & 5.38 & 1.79 & -.13 & 5.31 & 1.55 & -.08 \\
\hline Hair color & 5.64 & 2.19 & -.61 & 5.58 & 2.05 & -.53 \\
\hline Face length & 5.20 & 1.50 & .24 & 5.04 & 1.27 & .26 \\
\hline Mouth size & 5.22 & 1.67 & -.19 & 5.21 & 1.35 & -.15 \\
\hline Eyebrow shape & 4.63 & 1.77 & .34 & 4.67 & 1.56 & .23 \\
\hline Face width & 5.40 & 1.60 & -.08 & 5.61 & 1.37 & .31 \\
\hline Cheek position & 5.50 & .80 & -.19 & 5.35 & .74 & -.63 \\
\hline Face shape & 4.67 & 1.13 & -.41 & 4.83 & 1.06 & -.12 \\
\hline Eye color & 4.98 & 2.19 & .18 & 4.82 & 2.04 & .26 \\
\hline
\end{tabular}

(close together-far apart), face shape (round-square), face width (narrow-wide), face length (short-long), nose size (small-large), mouth size (small-large), eye color (light-dark), hair length (shortlong), hair color (light-dark), eyebrow shape (thin-thick), and cheek position (low-high). After all the pictures had been rated on all attributes, the subjects then rated each picture for overall attractiveness.

\section{RESULTS}

Summary data from each condition are shown in Tables 1 and 2 . For the purpose of the correlation-based analyses presented below, two points should be kept in mind. First, in each sample, the measures show sufficient variation to support an investigation of their patterns of covariation. That is, there is no apparent range restriction. Second, none of the measures in any sample are highly skewed. Thus, we can expect the correlation patterns within each sample (the bases for the factor analyses) to reflect the bivariate relationships among the variables, rather than to be influenced by properties of the univariate distributions.

Table 3 shows the correlations between the male and female ratings of each attribute, taken separately for each set of 40 same-gender pictures. These correlations are uniformly large, indicating very similar overall attribute perceptions by males and females.

The attribute intercorrelations from the four conditions were submitted to separate principal components analyses. Each of the analyses yielded a five-factor solution (as indicated by both "scree" analyses and the common criterion of $\lambda<1$ ), which accounted for about $76 \%$ of the total variance.

Table 4 shows the salient variables (using a cutoff of .3) for the five rotated components from each condition. Varimax solutions are shown; all other orthogonal and oblique rotations produced equivalent results, suggesting a very strong and stable structure to the data from each condition. Inspection of Table 4 reveals that although there is a strong general similarity among the solutions from the four conditions, there is greater similarity between conditions that involve the same picture gender.

The factors both for males rating males (MrM) and for females rating males (FrM) were: (1) face shape (consisting of face shape, face width, face length, and eye color); (2) eye (consisting of eye size and eye spacing); (3) smile (consisting of mouth size and cheek position); (4) hair (consisting of hair length and hair color); and (5) nose-brow (consisting of nose size and eyebrow shape).

The factors for females rating females (FrF) and males rating females $(\mathrm{MrF})$ were: (1) face shape (consisting of face shape, face length, and face width); (2) smile (consisting of nose size, mouth size, and cheek position, with female raters also including eye spacing); (3) coloration (consisting of hair color and eye color; (4) hair (consisting only of hair length for female raters; male raters also included eye spacing); and (5) eye structure (consisting of eye size and eyebrow shape).

The differences between the pairs of solutions were as follows: (1) Raters considering male pictures included eye color with the face shape factor, whereas raters of female pictures did not; (2) the smile factor consisted of more attributes for female pictures (mouth size, nose size, cheek position, and eye spacing) than it did for male pictures (nose size and cheek position); (3) raters considering male pictures seemed to group together aspects of hair (color and length), whereas for female pictures raters looked for

Table 3

Correlations between Mean Male and Female Ratings for Each Attribute

\begin{tabular}{lcccccccccccc}
\hline & \multicolumn{10}{c}{ Attributes } \\
\cline { 2 - 14 } Picture & $\begin{array}{c}\text { Hair } \\
\text { Gender }\end{array}$ & $\begin{array}{c}\text { Length } \\
\text { Spacing }\end{array}$ & $\begin{array}{c}\text { Nose } \\
\text { Size }\end{array}$ & $\begin{array}{c}\text { Eye } \\
\text { Size }\end{array}$ & $\begin{array}{c}\text { Hair } \\
\text { Color }\end{array}$ & $\begin{array}{c}\text { Face } \\
\text { Length }\end{array}$ & $\begin{array}{c}\text { Mouth } \\
\text { Size }\end{array}$ & $\begin{array}{c}\text { Eyebrow } \\
\text { Shape }\end{array}$ & $\begin{array}{c}\text { Face } \\
\text { Width }\end{array}$ & $\begin{array}{c}\text { Cheek } \\
\text { Position }\end{array}$ & $\begin{array}{c}\text { Face } \\
\text { Shape }\end{array}$ & $\begin{array}{c}\text { Eye } \\
\text { Color }\end{array}$ \\
\hline Male & .99 & .95 & .95 & .99 & .99 & .97 & .99 & .98 & .98 & .93 & .89 & .97 \\
Female & .98 & .95 & .97 & .98 & .92 & .96 & .98 & .98 & .99 & .85 & .92 & .99 \\
\hline
\end{tabular}


Table 4

Exploratory Factors from the Four Design Conditions

\begin{tabular}{lcccc}
\hline Face Shape & Smile & Nose-Brow & Eye & Hair \\
\hline $\begin{array}{l}\text { face width } \\
\text { face length } \\
\text { face shape } \\
\begin{array}{l}\text { eye color } \\
25.7\end{array}\end{array}$ & $\begin{array}{c}\text { mouth size } \\
\text { cheek position }\end{array}$ & $\begin{array}{c}\text { Males Rating Males } \\
\text { nose size } \\
\text { eyebrow shape }\end{array}$ & $\begin{array}{l}\text { eye size } \\
\text { eye spacing }\end{array}$ & $\begin{array}{l}\text { hair color } \\
\text { hair length }\end{array}$ \\
$\begin{array}{l}\text { face width } \\
\text { face length } \\
\text { face shape } \\
\text { eye color } \\
25.2\end{array}$ & $\begin{array}{l}\text { mouth size } \\
\text { cheek position }\end{array}$ & $\begin{array}{c}\text { Females Rating Males } \\
\text { nose size } \\
\text { eyebrow shape }\end{array}$ & $\begin{array}{l}\text { eye size } \\
\text { eye spacing }\end{array}$ & $\begin{array}{l}\text { hair color } \\
\text { hair length }\end{array}$ \\
& 14.5 & 14.8 & 10.8 & 8.5 \\
\end{tabular}

\begin{tabular}{|c|c|c|c|c|}
\hline Face Shape & Smile & Coloration & Hair & Eye Structure \\
\hline \multicolumn{5}{|c|}{ Females Rating Females } \\
\hline $\begin{array}{l}\text { face width } \\
\text { face shape } \\
\text { face length }\end{array}$ & $\begin{array}{l}\text { nose size } \\
\text { mouth size } \\
\text { cheek position } \\
\text { eye spacing }\end{array}$ & $\begin{array}{l}\text { hair color } \\
\text { eye color }\end{array}$ & hair length & $\begin{array}{l}\text { eye size } \\
\text { eyebrow shape }\end{array}$ \\
\hline 22.1 & 19.5 & 14.9 & 9.8 & 8.8 \\
\hline \multicolumn{5}{|c|}{ Males Rating Females } \\
\hline $\begin{array}{l}\text { face width } \\
\text { face shape } \\
\text { face length }\end{array}$ & $\begin{array}{l}\text { mouth size } \\
\text { cheek position } \\
\text { nose size }\end{array}$ & $\begin{array}{l}\text { hair color } \\
\text { eye color }\end{array}$ & $\begin{array}{l}\text { hair length } \\
\text { eye spacing }\end{array}$ & $\begin{array}{l}\text { eyebrow size } \\
\text { eyebrow shape }\end{array}$ \\
\hline $23.8^{\circ}$ & 17.7 & 15.1 & 8.7 & 9.8 \\
\hline
\end{tabular}

Note-Factors are arranged to show the similarity between the solution. The amount of variance accounted for by each factor is included beneath each factor.

coloration (hair and eye) and considered hair length a factor by itself; (4) raters looked at eye structure in female pictures (eye size and eyebrow shape), whereas for male pictures raters focused more on the eye itself (eye size and eye spacing), and (5) raters looked at the combination of nose size and eyebrow shape (nose-brow factor) in male pictures, but this was not the case for raters looking at female pictures.

Confirmatory techniques (oblique multiple groups component analyses, or OMG; see Gorsuch, 1983 and Bernstein, Garbin, \& Teng, 1988) were then employed to test how well the interpreted factors discussed above fit the data, and to test the patterns of similarities and differences among the four solutions.

For the first set of confirmatory analyses, the variance accounted for by the five-component solution was compared to the variance accounted for by the five-factor salient model, in which the factors were composed only of the variables with substantial loadings. Table 5 shows an example hypothesis matrix for a salient model. To help interpret the variance accounted for by the salient models, pseudofactors are constructed by randomly assigning variables to factors (matching the salient factors and pseudofactors as to the number of variables loading on each). These pseudofactors provide a worst case for the variance accounted for by that many components. If the interpretation is accurate, the salient model will account for about the same amount of variance as the full components (although for mathematical reasons the full components will always account for somewhat more variance), and it will account for substantially more variance than the pseudofactors. The first two rows and the last row of each division in Table 6 show the variance accounted for by five principal components, five salient factors, and the

Table 5

Hypothesis Matrix for Salient Factors for Female-Rating-Female Solution

\begin{tabular}{|c|c|c|c|c|c|c|c|c|c|c|c|c|}
\hline \multirow[b]{2}{*}{ Factor } & \multicolumn{12}{|c|}{ Attributes } \\
\hline & $\begin{array}{c}\text { Hair } \\
\text { Length }\end{array}$ & $\begin{array}{c}\text { Eye } \\
\text { Spacing }\end{array}$ & $\begin{array}{l}\text { Nose } \\
\text { Size }\end{array}$ & $\begin{array}{l}\text { Eye } \\
\text { Size }\end{array}$ & $\begin{array}{l}\text { Hair } \\
\text { Color }\end{array}$ & $\begin{array}{c}\text { Face } \\
\text { Length }\end{array}$ & $\begin{array}{c}\text { Mouth } \\
\text { Size }\end{array}$ & $\begin{array}{c}\text { Eyebrow } \\
\text { Shape }\end{array}$ & $\begin{array}{c}\text { Face } \\
\text { Width }\end{array}$ & $\begin{array}{c}\text { Cheek } \\
\text { Position }\end{array}$ & $\begin{array}{c}\text { Face } \\
\text { Shape }\end{array}$ & $\begin{array}{c}\text { Eye } \\
\text { Color }\end{array}$ \\
\hline 1 & 0 & 0 & 0 & 0 & 0 & +1 & 0 & 0 & -1 & 0 & +1 & 0 \\
\hline 2 & 0 & +1 & +1 & 0 & 0 & 0 & +1 & 0 & 0 & +1 & 0 & 0 \\
\hline 3 & 0 & 0 & 0 & 0 & +1 & 0 & 0 & 0 & 0 & 0 & 0 & +1 \\
\hline 4 & +1 & 0 & 0 & 0 & 0 & 0 & 0 & 0 & 0 & 0 & 0 & 0 \\
\hline 5 & 0 & 0 & 0 & +1 & 0 & 0 & 0 & +1 & 0 & 0 & 0 & 0 \\
\hline
\end{tabular}


Table 6

Comparison of Confirmatory and Exploratory Factor Solutions

\begin{tabular}{|c|c|c|c|}
\hline Source & h2 & $\begin{array}{c}\mathrm{Phi} \\
\text { Maximum }\end{array}$ & $\begin{array}{c}\text { Phi } \\
\text { Average }\end{array}$ \\
\hline \multicolumn{4}{|c|}{ Females Rating Males } \\
\hline FrM 5 PCS & 75.7 & & \\
\hline 5 HYP PCS & 73.7 & .32 & .14 \\
\hline FrM-MrM 5 PC cross & 75.4 & .07 & .02 \\
\hline FrM-FrF 5 PC cross & 67.2 & .36 & .18 \\
\hline $\begin{array}{l}\text { FrM-MrF } 5 \text { PC cross } \\
5 \text { PC pseudo }\end{array}$ & $\begin{array}{l}68.5 \\
60.5\end{array}$ & .35 & .21 \\
\hline \multicolumn{4}{|c|}{ Males Rating Males } \\
\hline MrM 5 PCS & 77.7 & & \\
\hline 5 HYP PCS & 75.2 & .29 & .15 \\
\hline MrM-FrM 5 PC cross & 77.2 & .11 & .04 \\
\hline MrM-FrF 5 PC cross & 66.9 & .40 & .19 \\
\hline MrM-MrF 5 PC cross & 68.6 & .39 & .21 \\
\hline 5 PC pseudo & 61.7 & & \\
\hline \multicolumn{4}{|c|}{ Males Rating Females } \\
\hline MrF 5PCS & 75.1 & & \\
\hline 5 HYP PCS & 71.5 & .26 & .15 \\
\hline MrF-FrF 5 PC cross & 74.2 & .21 & .06 \\
\hline MrF-MrM 5 PC cross & 63.4 & .38 & .19 \\
\hline MrF-FrM 5 PC cross & 65.0 & .43 & .19 \\
\hline 5 PC pseudo & 61.8 & & \\
\hline \multicolumn{4}{|c|}{ Females Rating Males } \\
\hline FrF 5 PCS & 75.1 & & \\
\hline 5 HYP PCS & 72.3 & .26 & 13 \\
\hline FrF-MrF 5 PC cross & 74.9 & .24 & .05 \\
\hline FrF-MrM 5 PC cross & 63.0 & .44 & .18 \\
\hline FrF-FrM 5 PC cross & 64.7 & .45 & .17 \\
\hline 5 PC pseudo & 60.7 & & \\
\hline
\end{tabular}

Note-phi maximum $=$ largest interfactor correlation; phi average $=$ average interfactor correlation. $\mathrm{FrF}=$ females rating females; $\mathrm{MrF}=$ males rating females; $\mathrm{MrM}=$ males rating males; $\mathrm{FrM}=$ females rating males. PCS $=$ exploratory principal components solution; $\mathrm{PC}=$ principal components; Hyp PC = hypothesized/salient principal components solution.

pseudofactors, respectively. In each case, the salient model accounted for nearly as much variance as did the five components and substantially more variance than did the five pseudofactors, indicating that the interpretations given above embody the important aspects of the factor solutions.

A second set of confirmatory analyses was used to test the notion, discussed above, that the MrM and FrM solutions share one structure that is different from the structure shared by the MrF and FrF solutions. The most direct method available to compare the factor structure of two (or more) solutions is to conduct a cross comparison, which is similar to the more common double cross validation used in regression analyses (see, e.g., Pedhazur, 1982). Each analysis begins with the correlation matrix from one solution and a hypothesis matrix, which is the factor score coefficient matrix from some comparison solution. (These analyses are conducted in pairs, with the factor score coefficient matrix from, say, the MrM solution applied to the FrM correlation matrix and, vice versa, the factor score coefficients of FrM applied to the MrM correlation matrix). If the two solutions have the same structure, the variance accounted for by the crossed analysis will be nearly as large as that accounted for by the exploratory principal components anal$y$ sis. If the two solutions have different structures, there will be a substantial difference in the variance accounted for by the component and crossed solutions. (It may be helpful to understand that when the factor score coefficient matrix from a solution is applied in an OMG analysis to the correlation matrix from which it was derived, the component and OMG structures will be exactly the same.)

Table 6 shows the results of the 12 cross comparisons. For each solution, the cross with the solution having the same picture gender (i.e., MrM-FrM and FrF-MrF) accounted for nearly as much variance as did the component solution (the largest drop was $0.9 \%$ of the variance). Also for each solution, crosses with the solutions with the opposite picture gender (e.g., FrM with FrF) produced substantial decreases in the variance accounted for (a range of $7.2 \%-12.1 \%$, with a median of $10.3 \%$ ). Taken together, the two sets of confirmatory analyses provide strong, direct support for the interpretation of each of the four component solutions and for the pattern of similarities and differences among the solutions.

The results of the exploratory and confirmatory factor analyses provide converging evidence that two different factor structures exist, and that they define the perceived patterns of interrelationships among the 12 facial attributes as suggested by the third hypothesis. These two patterns of interrelationships are related to the gender of the face being rated. The five factors for male and female subjects rating female faces were identified as face shape, smile, coloration, eye structure, and hair. The five factors for male and female subjects rating male faces were identified as face shape, smile, eye, hair, and nose-brow.

Correlation and regression analyses were restricted to the $\mathrm{MrF}$ and FrM groups, since they are the typical groups of interest in the study of attractiveness perceptions and mate selection. As for the factor analyses, the data for these correlations were aggregated across subjects to form an average value of the rated facial attributes and overall attractiveness for each picture, separately for the malesrating-females and females-rating-males data sets. The correlations were obtained by correlating these aggregate values across the 40 pictures.

Inspection of Table 7 reveals a mixture of similarities and differences among the attributes that are related to attractiveness for male and female raters. Hair length and face shape are correlates for both, with each group of raters preferring shorter hair and faces that tend to be more square. Other correlates for male raters are nose size (with smaller noses preferred), mouth size (smaller mouth preferred), and face width (preference for narrower faces). The other correlates for female raters are face length (longer faces preferred) and cheek position (higher cheeks preferred).

Table 8 shows the correlations between the face attribute factors described earlier and attractiveness. Only the face shape factor for FrM was significant. This is an interesting finding, for it suggests that although there are 
Table 7

Correlations of Attributes with Attractiveness for Males Rating Females and Females Rating Males

\begin{tabular}{lcc}
\hline \multicolumn{1}{c}{ Attributes } & MrF & FrM \\
\hline Hair length & $-.35^{*}$ & $-.31^{*}$ \\
Eye spacing & .05 & -.29 \\
Nose size & $-.39^{*}$ & -.06 \\
Eye size & -.12 & .05 \\
Hair color & -.03 & .06 \\
Face length & -.10 & $.41^{*}$ \\
Mouth size & $-.33^{*}$ & .12 \\
Eyebrow shape & -.07 & .02 \\
Face width & $-.35^{*}$ & -.21 \\
Cheek position & -.06 & $.35^{*}$ \\
Face shape & $.35^{*}$ & $.48^{*}$ \\
Eye color & -.12 & .01 \\
\hline
\end{tabular}

Note $-\mathrm{MrF}=$ males rating females, FrM $=$ females rating males. *Significant at $p<.05$, on the basis of a one-tailed significance test $(d f=38)$.

strongly perceived patterns of attribute correlations (i.e., the factors), these factors are not perceptual units with respect to the perception of attractiveness.

Multiple regression analyses revealed little additional information. In general, these analyses supported the interpretation of the pattern of simple correlations given above (e.g., which variables were and which ones were not related to attractiveness) and pointed out the considerable collinearity among the attributes, which was established in the earlier factor analyses. One interesting finding was that, for the males rating females, within a model that included all of the attributes, eye size acted as a significant suppressor variable (i.e., it had a small negative simple correlation but a large significant positive partial regression weight), probably because of its collinearity with mouth size, eyebrow shape, and face shape.

\section{DISCUSSION}

The high correlations resulting from a comparison of male and female ratings of each attribute (taken separately for each set of 40 same-gender pictures) indicate that males and females have very similar attribute perceptions. The apparent similarity of perceptions of attributes allows for an easier and more interpretable analysis of perceived patterns of interrelationships among the attributes. Ex-

\section{Table 8}

Correlations of Attribute Factors with Attractiveness for Males Rating Females and Females Rating Males

\begin{tabular}{lrc}
\hline \multirow{2}{*}{ Attribute Factors } & \multicolumn{2}{c}{$r$} \\
\cline { 2 - 3 } & MrF & FrM \\
\hline Face Shape* & .23 & $.43 \dagger$ \\
Eye (FrM only) & & .25 \\
Smile* & -.29 & .04 \\
Coloration (MrF only) & -.09 & \\
Hair* & -.27 & -.02 \\
Eye structure (MrF only) & -.12 & \\
Nose-brow (FrM only) & & .04 \\
\hline
\end{tabular}

Note- $\mathrm{MrF}=$ males rating females, $\mathrm{FrM}=$ females rating males. * See Table 3 for factor composition differences. +Significant at $p<.05$, on the basis of a one-tailed significance test $(d f=38)$. ploratory and confirmatory factor analyses indicated that males and females share similar perceived patterns of interrelationships among the 12 attributes and that there are two different patterns that depend on the gender of the face being judged. That is, male and female raters appear to have the same patterns of perceived interrelationships among the 12 attributes for female faces and they appear to agree on a different pattern of perceived interrelationships among the 12 attributes for male faces. The data support the hypothesis that the interrelationships among facial features depend on the gender of the face being evaluated, not on the gender of the rater. The resulting factors for male and female raters judging female pictures consisted of the following: a face shape factor consisting of face length, face shape, and face width; a smile factor consisting of mouth size, cheek position, and nose size (female raters also included eye spacing); a coloration factor consisting of hair color and eye color; a hair length factor (males included eye spacing); and the final factor, eye structure, consisting of combined eye size and eyebrow shape. The factors are somewhat similar for rating male pictures: a face shape factor consisting of face length, face shape, face width, and eye color; an eye factor consisting of eye size and eye spacing; a smile factor consisting of mouth size and cheek position; a hair factor consisting of hair length and hair color; and a nose-brow factor consisting of nose size and eyebrow shape. To our knowledge, this is the first study to show how people interrelate facial features, and it is the first to show that these interrelations are different for female faces as opposed to male faces.

Correlational and regression analyses revealed that although patterns of attribute interrelationships do exist for raters, these factors do not appear to be perceptual units in the perception of attractiveness. The attributes that were related to attractiveness were somewhat different for male as opposed to female raters. Both raters preferred shorter hair (possibly an indication of the hair trends of 1987 and 1988) and squarish (rather than round) faces, with males also preferring small noses, small mouths, and narrow faces. Females preferred longer faces and high cheeks.

The results of the present simple and multiple correlations based on attribute ratings share both similarities and differences with the findings of Cunningham (1986). Cunningham found that physical measures of eye height, eye width, eye separation, chin length, cheekbone width, eyebrow height, and pupil width were significantly correlated with attractiveness ratings for females. However, we found no correlations between perceptual ratings of eye size, eye spacing, face length, or eyebrow shape and attractiveness for males rating females. The present study supported Cunningham's findings that nose width and nose area (represented here as nose size) and cheek width (represented here as face width) are significant predictors of attractiveness, with male raters preferring smaller noses and narrow faces. In contrast to Cunningham's finding that males preferred a wide smile, the present study found that males preferred smaller mouths. 
We proposed that, in order to look at attractiveness ratings and the "rules" used by various persons to assign the rating, it would be helpful to establish that people perceive the physical facial attributes in the same manner, and that they have the same perceived patterns of interrelationships among the attributes. If these are established, the examination of the similarities among the "rules" becomes somewhat easier. The results of the present study support these two proposals and allowed for the analysis of the "rules" that people use to determine a stimulus person's facial attractiveness (i.e., which of the facial attributes were important to the assessment of facial attractiveness). The multiple regression analyses of the attractiveness ratings revealed that the "rules" used to determine facial attractiveness do not coincide with the perceived patterns of interrelationships revealed by the factor analysis, but "rules"' do seem to exist. However, with the exception of two facial attributes (hair length and face length), the "rules" are different for female stimulus faces and male stimulus faces.

\section{REFERENCES}

Berscheid, E., \& Walster, E. (1974). Physical attractiveness. In L. Berkowitz (Ed.), Advances in experimental social psychology (Vol. 7, pp. 158-216). New York: Academic Press.
Bernstein, I. H., Garbin, C. P., \& Teng, G. K. (1988). Applied multivariate analysis. New York: Springer-Verlag.

Bernstein, I. H., Lin, T.-D., \& McClellan, P. (1982). Cross- vs. within-racial judgments of attractiveness. Perception \& Psychophysics, 32, 495-503.

Buss, D. M. (1985). Human mate selection. American Scientist, 73, 47-51.

Cunningham, M. R. (1986). Measuring the physical in physical attractiveness: Quasi-experiments on the sociobiology of female facial beauty. Journal of Personality and Social Psychology, 50, 925-935.

GorsuCh, R. L. (1983). Factor analysis. Hillsdale, NJ: Erlbaum.

Malpass, R. S., \& Kravitz, J. (1969). Recognition of faces of own and other races. Journal of Personality \& Social Psychology, 13, 330-334.

Monahan, J. S., \& Lockhead, G. R. (1970). Identification of integral stimuli. Joumal of Experimental Psychology: General, 106, 94-110.

MuRstein, B. I. (1972). Physical attractiveness and marital choice. Journal of Personality \& Social Psychology, 22, 8-12.

Pedhazur, E. J. (1982). Multiple regression in behavioral research. New York: Holt, Rinehart \& Winston.

Shepherd, J. W., Deregowski, J. B., \& Ellis, H. D. (1974). A crosscultural study of recognition memory for faces. International Journal of Psychology, 9, 205-211.

Walster, E., Aronson, V., Abrahams, D., \& Rottman, L. (1966). Importance of physical attractiveness in dating behavior. Journal of Personality \& Social Psychology, 4, 508-516.

(Manuscript received July 3, 1989; revision accepted for publication March 29, 1990.) 